\title{
Online Monitoring of Hard Facing Coating on Stainless Steel Substrate using Infrared Thermography
}

\author{
Santosh. $\mathrm{S}^{1, \mathrm{a}}$, Rajkumar. $\mathrm{K}^{2, \mathrm{~b}}$, Menaka. $\mathrm{M}^{3, \mathrm{c}^{\star}}$, Venkatraman. $\mathrm{B}^{4, \mathrm{~d}}$ \\ ${ }^{1}$ PG Scholar, Department of Mechanical Engineering, SSN College of engineering, \\ Kalavakkam-603110, India \\ ${ }^{2}$ Associate Professor, Department of Mechanical Engineering, SSN College of engineering, \\ Kalavakkam-603110, India \\ ${ }^{3}$ Quality Assurance Division, Indira Gandhi Centre for atomic Research, Kalpakkam - 603 102, India \\ ${ }^{4}$ Radiological Safety and Environmental Group, Indira Gandhi Centre for atomic Research, \\ Kalpakkam - 603 102, India \\ asanth.2591@gmail.com, ${ }^{b}$ rajkumark@ssn.edu.in, ${ }^{c}$ menaka@igcar.gov.in, dbvenkat@igcar.gov.in
}

\begin{abstract}
Nuclear components such as grid plate and diverse safety rod drive mechanism work under high temperature service conditions and are subjected to severe wear and tear. Hard facing alloys like colmonoy and stellite are overlaid on such critical nuclear components using Gas Tungsten Arc Deposition techniques in order to with stand such adverse conditions. Stellite, a cobalt based alloy is not preferable for nuclear applications because, the cobalt wear particles and corrosion debris might get transformed into cobalt-60 by transmutation in the reactor, creating serious problems for the safety of nuclear plants owing to its long half-life. Among Nickel based alloys, Colmonoy grades are a possible replacement for Cobalt based Stellite alloys which have comparable hardness. Multi pass welding technique is employed for deposition of colmonoy on the stainless steel substrate. During deposition hot cracks tend to occur which are undesirable and weaken the coating. Therefore, characterization of such defects becomes very vital. Conventionally, these defects are characterized only after the welding process, by the use of NDT techniques. As a result the material may get rejected if any defects are encountered. In order to overcome this limitation, a real-time monitoring using infra-red thermography during gas tungsten arc deposition technique on stainless steel substrate has been attempted in this paper. Online images were processed and the cracks were mapped in order to determine the crack dimensions. Investigation of results authenticates that IR method can identify hot cracks online.
\end{abstract}

Keywords: Infrared Thermal Imaging, Online Monitoring, Hard Facing Coating, Hot cracks 